\title{
Preclinical Study of Morinda Citrifolia Linn Concentration and Usage Time and its Liver and Renal Toxicity
}

\author{
Ana Laura Carvalho Achkar, Caroline Garcia Francisco, Gabriela de Marco Cuginotti, Maria \\ Fernanda Ribeiro Cury, Luiz Gabriel Milanez Ronchi, Luiz Sergio Milanez Ronchi, Rafael de \\ Oliveira Guena., Thalyta Aparecida Leite de Lima, Carla Patrícia Carlos, Patrícia da Silva \\ Fucuta, Patrícia Maluf Cury, Tamara Veiga Faria" \\ Faceres medice school - Avenida Anísio Haddad number 6751, São José do Rio Preto, São Paulo, Brazil. \\ *Corresponding Author: Tamara Veiga Faria, Faceres medice school - Avenida Anísio Haddad number \\ 6751, São José do Rio Preto, São Paulo, Brazil.
}

\begin{abstract}
The popularity of Morinda Citrifolia Linn (Noni) as a dietary supplement, a food functional ingredient, or as a natural health enhancer is increasing throughout the world. Our study aims to investigate the concentrations of $10 \%$ and $50 \%$ of Morinda Citifolia Linn and usage time and its renal and hepatic toxicity. To this purpose, 42 male wistar rats were distributed into groups: treated for 15 days with concentration of $10 \%$ and 50 and treated for 90 days with concentration of $10 \%$ and $50 \%$. Noni juice has been prepared with fruit's pulp and seeds at concentrations of 10\% and 50\% diluted in water. The groups 15 and 90 days presented elevated creatinine, urea, AST and ALT, compared to respectively control groups $(p<0,05)$, and the levels of these markers are higher in 90 days groups. The presence of steatosis and dermatological changes was observed in rats in 90-days and concentration 50\%. The fruit juice of Morinda Citrifolia Linn at concentration of 50\% causes damage to liver and kidney function, being able to induce development of non-alcoholic liver disease, regardless of usage time. The alterations of hepatic and renal markers are directly proportional to usage time and dose-concentration.
\end{abstract}

Keywords: Experimental study; Morinda Citrifolia Linn; Hepatotoxicity; Nephrotoxicity; Toxicidade;

Abbreviations: Noni: Morinda Citrifolia Linn, AST: aspartate aminiotranferase, ALT: alamine aminiotranferase, C15: group of animals treated for 15 days with water, C90: group of animals treated for 90 days with water, N15Min: group of animals treated for 15 days with Noni in concentration of 10\%, N15Max: group of animals treated for 15 days with Noni in concentration of 50\%, N90Min: group of animals treated for 90 days with Noni in concentration of 10\%, N90Max: group of animals treated for 90 days with Noni in concentration of $50 \%$.

\section{INTRODUCTION}

Morinda Citrifolia Linn, a fruit belonging to the Rubiaceae family, native to Southeast Asia (Indonesia) and Australia, also known as Ba Ji Tian, Nonu, Indian Mulberry, Canary wood, popularly known as Noni, is widely used for treatment of several diseases and gradually became prominence by its numerous medical benefits reported ${ }^{[1,2]}$. It is an ovoid-shaped fruit, succulent and contains many triangular seeds of reddish coloration ${ }^{[3]}$ and have 160 different phytochemicals. Because is easily accessible and due to its possible therapeutic actions, it became popular ${ }^{[4-6]}$. Among its compounds, anthraquinones (damnacanthal, morindona, morindine) are notable because may have action in several cellular and molecular factors resulting in their various therapeutic outcomes ${ }^{[7]}$.

However, despite its popular use for more than 2000 years in Asia, few studies have been able to showed effectively its real therapeutic benefits such as antimicrobial, analgesic ${ }^{[8]}$, anti-inflammatory ${ }^{[8]}$, antidiabetic ${ }^{[7,8]}$, anticancer, antioxidant, immunostimulant activity, healing ${ }^{[2,5]}$ and anti-angiogenic activity ${ }^{[2]}$.

Meanwhile, even by popular reports and scientific literature review describing its benefits, studies have revealed that fruit consumption may be related to hepatotoxicity and nephrotoxicity cases, requiring caution in its use ${ }^{[9]}$. In 2005, researchers reported the possibility of Noni being hepatoxic ${ }^{[9]}$, and other related a relationship between Morinda Citrifolia Linn and acute idiosyncratic hepatitis and the possibility of causing liver and kidney damage ${ }^{[9-12]}$. 
It is well known that medicinal plants can generate compounds that can be used as therapeutic agents. Thus, it is essential to evaluate its effectiveness by setting its safety and dose-response relationship.

In this rational, Noni, could be subject to pre-clinical and clinical studies due to its popularity, easy access, low cost and possible therapeutic benefits in many diseases. Our study aims to investigate the concentrations of $10 \%$ and 50\% of Morinda Citifolia Linn and usage time and its renal and hepatic toxicity.

\section{MATERIALS AND MeTHOdS}

\subsection{Animals}

All experimental procedures were conducted according to the guidelines for biomedical research stated by the Brazilian Societies of Experimental Biology and approved by the Ethics Committee on Animal Experimentation of FACERES School of Medicine with protocol number 007/2016. Wistar male rats were allocated in individual cages, remaining in a controlled environment with temperature of $22^{\circ} \pm 2$ ${ }^{\circ} \mathrm{C}$, relative air humidity at $60 \%$ and 12 hours light-dark cycle. All animals received same ad libitum feeding conditions with a standard balanced rodent feed (Labina, Purina). 42 male Wistar rats weighing 200 to $240 \mathrm{~g}$ were randomly distributed in two groups: group $C$ (Control) and group N (Noni).

Group C, with 14 rats, was divided into C15 and C90 groups (7animals per group) and received water and feed consumption evaluated daily. They were sacrificed at 15 days (C15) or 90 days (C90), respectively. Group N, with 28 Noni juice-treated animals, was also divided according to treatment time and juice concentration (7 animals per group) being: N15Min and N15Max (treated for 15 days with concentration of $10 \%$ and 50\%, respectively), and N90Min and N90Max (treated for 90 days with concentration of $10 \%$ and $50 \%$, respectively).

\subsection{Preparation of Investigational Product}

The fruits of Morinda Citrifolia Linn, Rubiaceae family, popularly known as Noni were acquired in São José do Rio Preto (SP, Brazil). They were selected according to the whitish yellow aspect, which is equivalent to their maturity. After washing in running water, the whole fruits were placed into centrifuge and the juice was prepared with no addition of vehicle, sugar or sweetener. From a homogeneous mixture and weighing, concentrations of $10 \%$ and $50 \%$ were prepared with addition of mineral water.

\subsection{Procedures}

Daily, the animals received $300 \mathrm{ml}$ of Noni juice at $10 \%$ and $50 \%$ concentrations with measuring the amount ingested by both groups. Weekly, they had undergone to physical evaluation for checking their weight, behavioural changes, pelage, and cutaneous alterations.

The sacrificial procedures were performed on $16^{\text {th }}$ or $91^{\text {st }}$ treatment days by an anesthetic overdose (20 $\mathrm{mg} / \mathrm{kg}$ of Xylazine Hydrochloride and $170 \mathrm{mg} / \mathrm{kg}$ of intraperitoneal Ketamine). A laparotomy was performed for macroscopical inspection of abdominal cavity and organs, and blood sample was collection through cava vein. The samples were centrifuged and plasma used for determination of transaminases, creatinine and urea by colorimetric assay (spectrophotometry, BIO-200S, Bioplus, São Paulo, SP, Brazil) by commercial kit (Biotechnical, Varginha, MG, Brazil). Liver and kidneys were removed and fixed in $4 \%$ paraformaldehyde, sodium phosphate buffer $0.1 \mathrm{M}, \mathrm{pH} 7.4$, for 24 hours at $4^{\circ} \mathrm{C}$, transferred to an alcohol $70^{\circ}$ solution and later embedded in paraffin. Sections of $5 \mu \mathrm{m}$ were submitted to staining by hematoxylin-eosin (HE) and Schiff's periodic acid (PAS).

We performed a semi quantitative analysis in a blinded fashion of histological slides. Liver histopathological analysis was evaluated by presence of necrosis, vascular congestion, steatosis and inflammation. Kidneys were evaluated by presence of glomerular sclerosis and inflammation. Five fields of each fragment were evaluated in $40 \mathrm{x}$ objective at optical microscopy (Primo Star, Zeiss). Analysis were conducted according to a score assigned as follows: 0 (no field affected), 1 (up to 25\% affected), 2 (26 to 50\% affected), 3 (51 to $75 \%$ affected) and 4 (76 to 100\% affected). Images were captured by camera attached to microscope and ZEN Lite software (Zeiss).

\subsection{Statistical Analysis}

Results were submitted previously to descriptive statistics and normal determination by KolmogorovSmirnov test for plasma comparisons we used Mann-Whitney test and Fisher's exact test was used to compare histopathological analysis results. A $p$ value of $\leq 0.05$ was considered significant. 


\section{RESULTS AND DISCUSSION}

Groups treated with Noni 50\% (N15Max) and 10\% (N15Min) for 15 days showed high plasma creatinine level, urea, aspartate aminiotranferase (AST) and alamine aminiotranferase (ALT), compared to control groups (C15). Histopathological analysis showed presence of no statistically significant structural changes. There were hydropic degeneration of renal tubules in $14.3 \%$ of N15Max group $(\mathrm{p}=$ $1.0) ; 14.3 \%$ of animals treated with Noni $10 \%$ showed liver steatosis $(p=1.0)$, and $14.3 \%$ showed nonspecific renal changes $(\mathrm{p}=1.0)$. Liver alterations were not found in N15Max and C15 groups, neither renal alterations in $\mathrm{C} 15$ group.

Similarly, groups treated for 15 days, animals treated with Noni $10 \%$ and $50 \%$ for 90 days also showed an increase in plasma creatinine, urea, AST and ALT. Comparative analysis between groups treated with Noni 10\% (N15Min and N90Min) showed higher plasma level of creatinine, urea, AST and ALT in animals treated for 90 days. Animals treated with Noni $50 \%$ for 90 days had only increase of creatinine and urea plasma level, compared to those treated for 15 days (Figure 1).
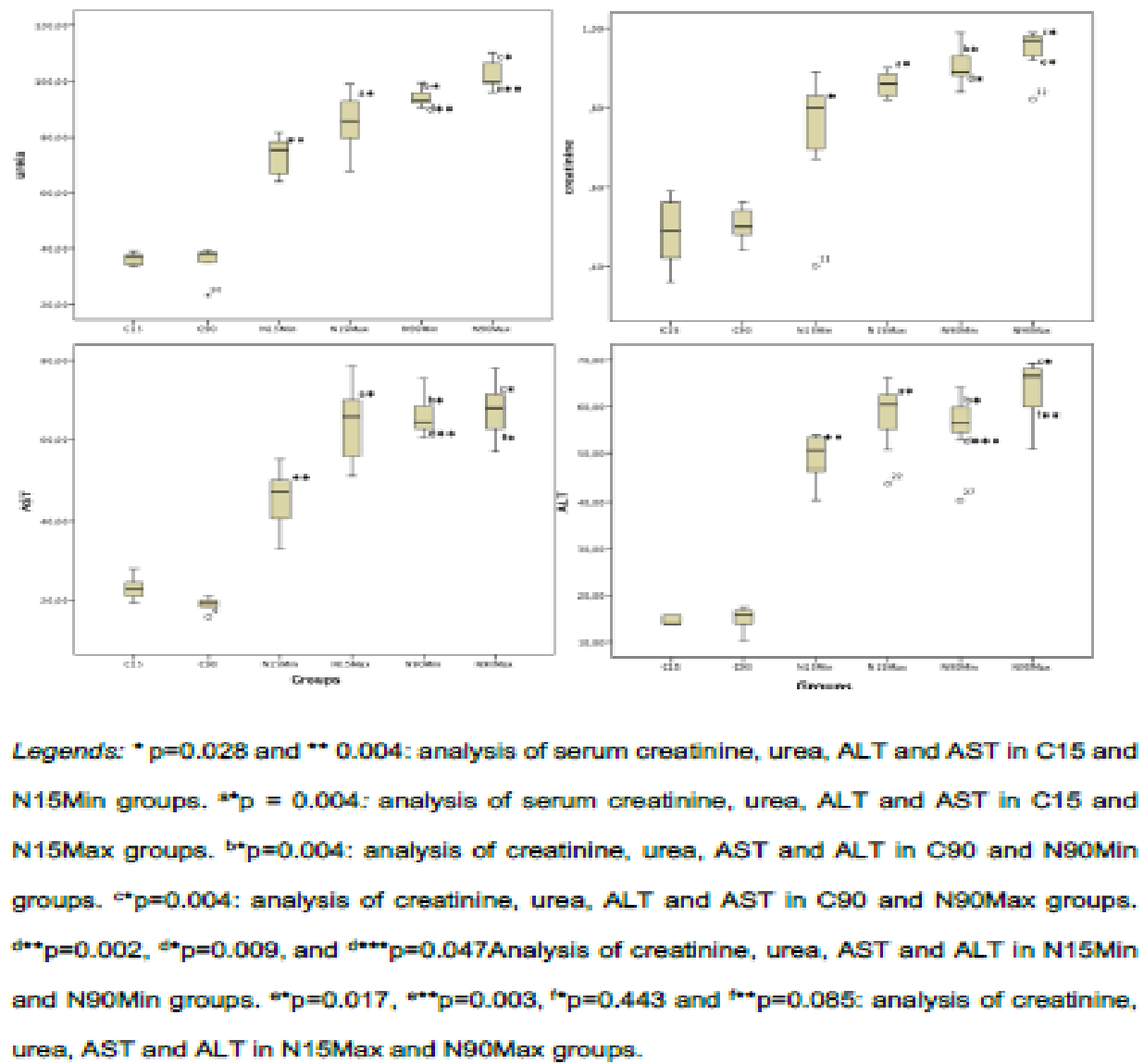

Figure1. Analysis of creatinine, urea, AST and ALT in groups.

This pre-clinical study shows a risk for liver and kidney toxicity due to Noni (Morinda Citrifolia Linn) fruit's juice given at concentrations of $10 \%$ and $50 \%$ to animals during 15 and 90 days. Treatment of animals with Noni juice, regardless of concentration methods (10\% and 50\%) and time of administration (15 and 90 days), caused increase of renal plasma level markers (creatinine and urea) and hepatic markers (ALT and AST). However, this deleterious effect is higher in the animals treated with dose of $50 \%$ in acute intoxication, which shows that damage of Morinda C. Linn to renal and hepatic systems can be dose-dependent in short term.

Regarding histopathologic alterations, $71.4 \%$ of N90Min group animals presented some hepatic changes $(\mathrm{p}=0.028)$. Among these alterations, $28.6 \%$ presented congestion $(\mathrm{p}=0.470), 57.1 \%$ steatosis $(\mathrm{p}=0.081)$, and $28.6 \%$ inflammatory infiltrate $(\mathrm{p}=0.470) .42 .9 \%$ showed non-significant renal alteration $(\mathrm{p}=0.205) .71 .4 \%(\mathrm{p}=0.028)$ of N90Max group animals showed liver steatosis and $42.9 \%$ $(\mathrm{p}=0.205)$ showed a nonspecific renal histopathological alteration. Control group did not present liver or renal alterations (Figure 1). 
The acute biochemical alterations of renal and hepatic markers caused by Noni were not accompanied by changes in the tissue structure of organs. The groups treated with two doses (10\% and 50\%) during 15 days showed no statistically significant histopathological alterations. However, hepatic histopathologic alterations are significant in animals treated with the highest dose (50\%) in long term (90 days), but not accompanied by changes on renal structure.

Animals from groups treated or not with Noni were fed daily with ad libitum and common feed and shared the same environment and care. There was no statistical difference regarding amount of feed ingested between groups, and due to that, presence of hepatic steatosis may not be related to feeding. Moreover, according to the current literature review, investigational product is composed of a large amount of carbohydrates and proteins and a small amount of lipids ${ }^{[2]}$, a fact that would not justify the hepatic steatosis identified in these animals. Therefore, results of this study with Morinda C. Linn, mainly at concentration of $50 \%$, are related to development of non-alcoholic fatty liver disease, accompanied by elevated transaminases. Our findings may contribute to explanation of hepatitis developed in patients using Noni in a case report ${ }^{[9]}$.

In the comparison between concentrations of $10 \%$ and $50 \%$ of Noni consumed during 90 days, it was found increased levels of creatinine, urea, AST, and ALT, higher in animals that ingested the concentration of $50 \%$. This finding corroborates with the hypothesis that elevation of hepatic transaminases is related to Noni administration and its concentration, accompanied by development of hepatic steatosis. The liver is main organ involved in biotransformation of any substance foreign to body, which makes it particularly vulnerable to chemical toxicity ${ }^{[10]}$. According to Stadlbauer, $2005^{[9]}$, who described a serial case report, there is a relationship between acute hepatitis, including indication of liver transplantation, to intake of 2 liters of Morinda C. Linn juice for 90 days. Although this author has not presented the concentration of Morinda C. Linn consumed during the 3 months by the patients, the data reinforce the idea that time and quantity of Noni intake increase risk of liver diseases.

A possible explanation for the hepatotoxic effect of Morinda C. Linn may be the presence of components such as rubidian, anthraquinones, damnacantal, and morindona. Anthraquinones produce free radicals derived from oxygen, which lead to depletion of intracellular glutathione and decrease of mitochondrial membrane potential, causing lipid peroxidation, oxidative stress and cell death ${ }^{[2]}$. In addition, the metabolism of proteins, carbohydrates, fat and drugs occurs in the liver, making this organ vulnerable to diseases. The use of medicinal plants for health promotion and as therapeutic target has caused hepatic diseases and increased number of steatosis cases ${ }^{[13]}$.

During data collection, it was observed skin lesion caused for cutaneous pruritus in the animals of group treated with 50\% Noni for 90 days. This observation was confirmed by skin histological analysis, which showed $85.7 \%$ of animals presenting dermatological inflammation $(\mathrm{p}=0.001)$, with presence of eosinophils $(28.6 \%)$, no significant $(\mathrm{p}=0.470)$ (Figure 1$)$.

In addition to hepatic findings, we emphasize that animals using Noni at concentration of $50 \%$ for 90 days, different from other groups, developed cutaneous lesions. Moreover, during data collection, animals also presented pruritus which may have contributed to exacerbate their cutaneous lesions. These alterations may be related to the rising of transaminases and to the risk of liver disease or possible hepatic damage caused by Morinda C. Linn administration.

According to Maia (2014) ${ }^{[13]}$, liver disease may be associated with several extrahepatic signs such as in the renal and nervous systems, gastrointestinal tract and skin. Cutaneous changes may represent the first sign of disease hepatic risk or impairment since $91.4 \%$ of patients with hepatic disease develop cutaneous manifestations ${ }^{[13]}$. Unlike our results and other study ${ }^{[9]}$, Serafini and collaborators (2011) ${ }^{[14]}$ showed that doses of $620 \mathrm{mg}$ ingested by Wistar rats, twice a day, $1000 \mathrm{mg} / \mathrm{day}$ or $30 \mathrm{ml} / \mathrm{day}$ can be considered safe because they are not lethal and do not cause cytotoxicity. However, these results do not imply that Morinda C. Linn would have no toxic effects because the authors used leaf extract ${ }^{[14]}$.

Comparison between 15 and 90 days groups with Noni intake in concentration of 10\% showed timedependent increased risk of liver and renal toxicity, represented by increase of biochemical serum markers (creatinine, urea, and transaminases), since at the same concentration, the use for 90 days showed higher hepatic toxicity. Furthermore, data related to concentration of $50 \%$ showed cumulative renal risks and consequently related to time, while liver damage can occur independently of time.

Lim (2016) ${ }^{[5]}$ studied the Noni leaf extract in BALC/c mice, comparing its action with the Erlotinib, an inhibitor of the epidermal growth factor receptor (EGFR) tyrosine kinase that is used in the treatment 
of non-small cell lung cancer, pancreatic cancer and several other types of cancer, which is already approved for treatment of lung cancer, due to improvement of therapeutic response of hepatic metastases of this malignant neoplasia. It was suggested that Noni's leaf extract might be a therapeutic option or a dietary supplement for prevention and management of inflammatory conditions of lung cancer, oxidative stress and tumoral development and metastases ${ }^{[5]}$. In this sense, it was demonstrated that damanacanthal, one of components of $M$. Citrifolia, has in vitro inhibitory action of tyrosine kinase receptor, which may act by inhibition of tumour angiogenesis and cell proliferation ${ }^{[5]}$.

The development of nephrotoxicity by Noni's use is sustained by the principle that kidneys are susceptible to cumulative and idiosyncratic effects from several drugs, and as well as these, elimination of compounds from the Noni fruit's metabolites ${ }^{[12]}$. The renal cells, by use of medicinal plants, become exposed to their compounds, which can cause nephrotoxicity ${ }^{[15]}$. Therefore, association of renal lesions with a long-term use of medicinal plants should be target of pre-clinical and clinical studies. Used as indicators of renal and glomerular function, serum levels of urea and creatinine, when increased, may precede renal lesions or represent organ damage ${ }^{[15]}$. In our study, these markers were raised in groups of animals whose received Noni at concentrations of $10 \%$ and $50 \%$. As on liver function, it was evidenced that concentration of $50 \%$ during prolonged period represents risks for renal function.

Renal function alterations were not accompanied by renal histopathological alterations. However, it is possible that the Noni causes damage to its structure by the use for a longer time. Histopathological analysis evidences incidence of structural alteration, that although not statistically significant at 90 days of Noni administration, may damage the organ for a longer period of time use. Reinforcing this assumption, we have the finding that levels of creatinine and urea are higher in the rats exposed for 90 days compared to those treated for 15 days, similarly to Noni's effect in the liver. A Brazilian study evaluating genotoxic activity of fruit demonstrated expressive damage to renal cells in animals of both genders treated during 14 days with concentrations of 5 and $10 \mathrm{mg} / \mathrm{Kg}$ of mature fruit ${ }^{[15]}$. Although our study has not evaluated genotoxicity, the concentrations studied were higher than those cited in the study, and consequently point to possible genotoxic damage.

Our data are in accordance with the study of Hadijah (2008) ${ }^{[16]}$, who analysed three concentrations Morinda C. Linn's fruit (5\%, 10\%, and 20\%) in Sprague-Dawley rats treated for 13 weeks. No red and white cell alterations were identified in the hemogram, but platelet counts were elevated, which could be related to possible hepatic damage. Moreover, there was also elevation of transaminases, urea and creatinine at concentration of $20 \%{ }^{[16]}$. However, unlike our data, authors considered safe the dosages of 5 and $10 \%$ of Morinda citrifolia Linn. mature fruits. Unlike the observed, in another study ${ }^{[15]}$, animals using Morinda C. Linn, at 3 different doses (10, 5, and $2.5 \mathrm{mg} / \mathrm{kg}$ of fruit) for 14 days, presented small elevation levels of urea and creatinine, not significant to establish renal injury according to the authors [15].

The comparison of acute use to chronic use of Noni at a concentration of $10 \%$ showed alterations in liver enzymes and renal markers. Therefore, the toxicity of Morinda C. Linn is not only a dependent dose as mentioned on a previous study ${ }^{[17]}$. There is also influence of exposure time. Moreover, according to Pola and collaborators, Noni has antioxidant activity when used under form of fruit powder, and consumption of small quantities provides greater benefit than large quantities ${ }^{[17]}$. Thus, concentration of $10 \%$, according to our data, could represent a study proposal about this antioxidant activity.

In short, the use of in natural phytotherapics may include substances not purified with unknown action and is subject to an absence of concentration control from these compounds, besides possibility of interaction between them ${ }^{[6]}$. This can cause toxicity, pathological alterations, and even death ${ }^{[4]}$. Despite numerous benefits reported popularly, clinical studies are still needed that may justify benefits of using Noni. The present study, by managing of fruit and seeds of M. Citrifolia, emphasizes that its intake is safe only with medical monitoring of liver and renal function, differently from protective effect observed on lung cancer by use of leaf extract ${ }^{[5]}$.

\section{CONCLUSION}

The fruit juice of Morinda Citrifolia Linn, Noni, at concentration of 50\%, causes damage to liver and kidney function, being able to induce development of non-alcoholic liver disease, regardless of usage time. We conclude that alterations of hepatic and renal markers are directly proportional to time of use 
and dose-concentration, and $10 \%$ dose is more indicated for establishment of Noni's therapeutic benefits, since it is accompanied by regular clinical monitoring.

\section{REFERENCES}

[1] Rodrigues da Silva L, Queiroz de Medeiros PV, Andrade Leite G, Pereira Silva KJ, Mendonça V, Galdino da Silva G. Caracterização do fruto de Morinda citrifolia L. (noni). Revista Cubana de Plantas Medicinales. 2012;17:93-100.

[2] Barbosa AF, Costa ICdM, Langassner SZ, Giordani RB. Morinda citrifolia: fatos e riscos sobre o uso do noni. Revista Fitos, Rio de Janeiro, 2017 ,Vol. 11(2), 119-249,

[3] Matoso LML, de Melo CCR, Menezes LMdCS, de Oliveira LE, de Oliveira KKD, As Características e a Utilização do Noni (Morinda Citrifolia).,C\&D-Revista eletronica de Fainor,, 2013, 42-50,6(1), 2013.

[4] CAMPOS S, SILVA C, CAMPANA P, ALMEIDA VJ. Toxicity of plant species. Rev. bras. plantas med. [online]. 2016, vol.18, p.373-382

[5] Lim SL, Mustapha NM, Goh YM, Bakar NA, Mohamed S. Metastasized lung cancer suppression by Morinda citrifolia (Noni) leaf compared to Erlotinib via anti-inflammatory, endogenous antioxidant responses and apoptotic gene activation. Mol and Cel biochem. 2016;416(1-2):85-97.

[6] Tovar RT, Petzel RM. Herbal toxicity; Dis Mon. 2009;55(10):592-641.

[7] Ruhomally Z, Somanah J, Bahorun T, Neergheen-Bhujun VS. Morinda citrifolia L. fruit extracts modulates H2O2-induced oxidative stress in human liposarcoma SW872 cells. Journal of traditional and complementary medicine. 2016;6(3):299-304.

[8] Assi RA, Darwis Y, Abdulbaqi IM, Vuanghao L, Laghari MJAJoC. Morinda citrifolia (Noni): A comprehensive review on its industrial uses, pharmacological activities, and clinical trials. 2017;10(5):691707.

[9] Stadlbauer V, Fickert P, Lackner C, Schmerlaib J, Krisper P, Trauner M, et al. Hepatotoxicity of NONI juice: report of two cases. World journal of gastroenterology. 2005;11(30):4758-60.

[10] García-Cortés M, Andrade R, Lucena M, González-Grande R, Camargo R, Fernández-Bonilla E, et al. Hepatotoxicidad secundaria a fármacos de uso común., Gastroenterol Hepatol, 2005;28(8):461-72.

[11] West BJ, Jensen CJ, Westendorf JJWJoGW. Noni juice is not hepatotoxic. World J Gastroenterol., 2006; 12(22): 3616-3619

[12] Naidoo S, Meyers AM. Drugs and the kidney.,SAMJ ,2015;105(4):332-7.

[13] Maia NC, Araújo FC, Pires CAA, Conde SRSdS, Nunes HM, Soares MdCP. Manifestações dermatológicas em uma casuística de doença hepática crônica de um hospital de referência em Belém, Estado do Pará, Brasil. Rev Pan-Amaz Saude 2014; 5(2):43-51

[14] Serafini MR, de Lima CM, Santos RC, Dória GAA, de Jesus JY, de Melo MGD, et al. Pre-clinical toxicity of Morinda citrifolia Linn. leaf extract., african journal of biotechnology 2011; vol 10(65):14566-72.

[15] Alencar MVOB, Silva MBS, Paz MFCJ, de Moraes GP, Nunes AT, Cavalcante AAdCM. Genotoxicidade e nefrotoxicidade da Morinda citrifolia em estudos pré-clínicos: riscos à saúde pública., R. Interd, 2013;6(1):1-8.

[16] Hadijah H, Normah A, Tarmizi SA, Thirteen-week toxicity study of mengkudu juice (Morinda citrifolia): Effect on the blood analysis., J. Trop. Agric. and Fd. Sc. ,2008;36(2):000-000.

[17] Pola CC, Junqueira M, Carvalho R, Teixeira LJQ, Saraiva SH. Efeito do processamento na capacidade antioxidante do noni (Morinda citrifolia). ENCICLOPÉDIA BIOSFERA, Centro Científico Conhecer ,2011;7(12):1-16.

Citation: Tamara Veiga Faria, et.al., (2019). Preclinical Study of Morinda Citrifolia Linn Concentration and Usage Time and its Liver and Renal Toxicity. International Journal of Medicinal Plants and Natural Products (IJMPNP), 5(4), pp.1-6. http://dx.doi.org/10.20431/2454-7999.0504001

Copyright: () 2019Authors, this is an open-access article distributed under the terms of the Creative Commons Attribution License, which permits unrestricted use, distribution, and reproduction in any medium, provided the original author and source are credited. 